\title{
Distribución del tomate de árbol Cyphomandra betacea (Cav.) Sendt. y caracterización eco-climática en las zonas del cultivo de Nariño
}

\section{Distribution of tree tomato Cyphomandra betacea (Cav.) Sendt. and the eco-climatic characterization of the areas of its cultivation in Nariño}
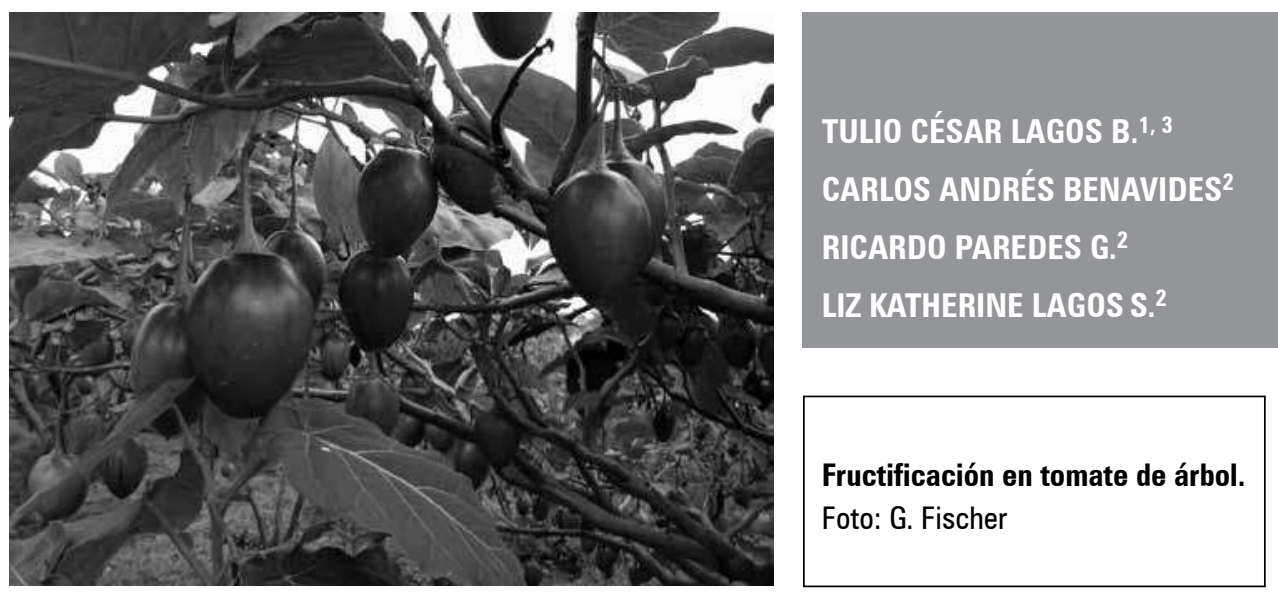

\section{RESUMEN}

La Universidad de Nariño cuenta con una colección de trabajo de 56 introducciones de tomate de árbol provenientes de la zona andina del departamento de Nariño. Esta presenta cierta variabilidad fenotípica, que depende del sitio de procedencia. La distribución general de Cyphomandra betacea en Nariño ha sido poco explorada, de ahí que la colecta de genotipos que aporten a la variabilidad de la colección, se puede ver limitada por el desconocimiento de dichas zonas. Esta investigación estuvo orientada a establecer la posible distribución de C. betacea en el departamento a partir del análisis de información de georreferenciación, así como a identificar las posibles zonas ecoclimáticas donde la especie se encuentra. La mayor probabilidad de distribución de la especie se encuentra en altitudes entre 1.500 y 3.000 msnm. Los grupos de distribución climática son dos: el grupo 1 con 35 accesiones y el grupo 2 con 21. Los dos grupos presentan similitud en cuanto a precipitación y temperatura, por lo que puede considerarse un clima templado húmedo mesotérmico. Se identificaron zonas con probabilidades de encontrar formas de C. betacea creciendo bajo condiciones climáticas similares a los grupos identificados en el análisis de agrupamiento del presente estudio. Existe una alta probabilidad de encontrar mayor variabilidad de C. betacea en los municipios de Pasto, Contadero, Cumbitara, Los Andes, San Pablo, Colón, Belén, La Unión, Albán, San Pedro de Cartago, Buesaco, Iles, Gualmatan, Pupiales, Ipiales, Funes, Puerres, Córdoba, Potosí, Santacruz, Túquerres, Tangua y Yacuanquer.

Facultad de Ciencias Agrícolas, Universidad de Nariño, Pasto (Colombia).

2 Facultad de Ciencias Agrícolas, Grupo de Investigación en Producción de Frutales Andinos (GPFA), Universidad de Nariño, Pasto (Colombia).

3 Autor para correspondencia.tclagosb@udenar.edu.co 


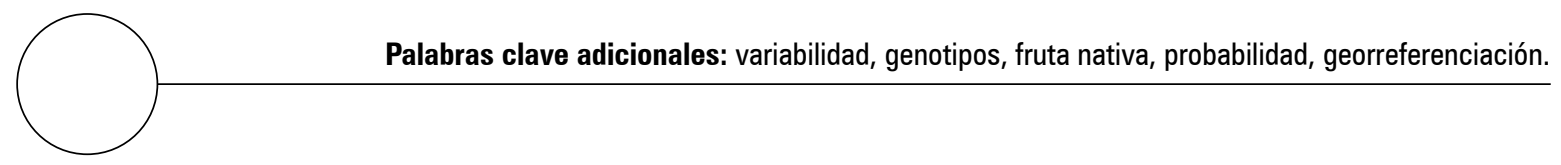

\section{ABSTRACT}

The University of Nariño has a work collection of 56 varieties of the tree tomato from the Andean region of Nariño, which demonstrates its phenotypic variability which is correlated to the site of origin. The overall distribution of Cyphomandra betacea in Nariño has been little explored; hence the collection of genotypes that contribute to the variability of the collection may be limited by unknown factors in those areas. This research was conducted to determine the possible distribution of $C$. betaceae in the region using an analysis of geographically referenced information, as well as, identifying potential eco-climatic zones where the species occurs. The most likely distribution of the species is found at altitudes between 1,500 and 3,000 m. There are two climatic distribution groups: group one with 35 varieties and group two with 21 . The two groups are similar in terms of precipitation and temperature, with climates which can be considered humid, mesothermic and mild. We identified areas where it is likely to find $C$. betaceae growing under climatic conditions similar to those of the groups identified in the cluster analysis of this study. There is a high probability of finding higher variability of $C$. betacea in the municipalities of Pasto, Contadero, Cumbitara, Los Andes, San Pablo, Colón, Bethlehem, La Unión, Albán, San Pedro de Cartago, Buesaco, Iles, Gualmatan, Pupiales, Ipiales, Funes, Puerres, Córdoba, Potosí, Santacruz, Túquerres, Tangua and Yacuanquer.

Additional key words: variability, genotypes, a native fruit, probability, georeferencing.

Fecha de recepción: 28-01-2011

Aprobado para publicación: 27-05-2011

$\longrightarrow$

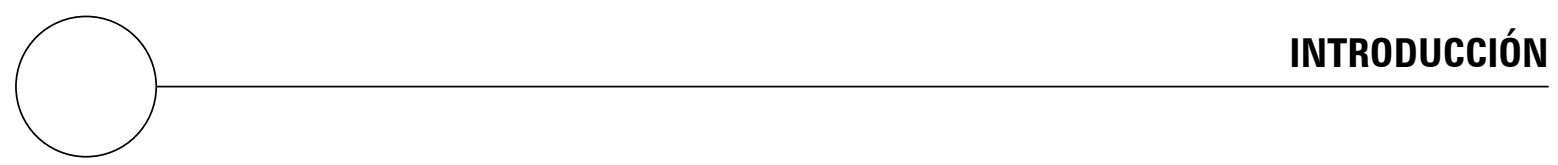

Bohs (1988) indica que el género Cyphomandra incluye alrededor de 50 especies que se encuentran en toda Suramérica hasta el sur de México, donde existen dos centros principales de diversidad, uno en el este de las laderas de los Andes del Perú y Bolivia y el otro en el sureste de Brasil. La mayoría son especies subtropicales que habitan en los bosques húmedos en altitudes a partir de 500 y hasta $2.000 \mathrm{msnm}$. Algunas especies se encuentran en la cuenca amazónica en elevaciones inferiores a los $100 \mathrm{msnm}$, y otras especies se encuentran en los bosques nublados hasta los
$3.000 \mathrm{msnm}$. Estas especies, generalmente son árboles de rápido crecimiento del sotobosque.

A pesar de ser el tomate de árbol originario de los Andes en América Latina, solamente es cultivado de manera extensiva en Ecuador y Colombia. Países como Nueva Zelanda, Kenia, Sri Lanka e India, son los principales productores a nivel mundial (Pringle y Murray, 1991). Según Lobo (2006), en Colombia existe una baja oferta en la variabilidad de los genotipos cultivados, ya que en la mayoría de los casos los agricultores selec- 
cionan sus propios genotipos, los cuales tienen una base genética estrecha.

La mayoría de las frutas andinas presentan un gran potencial para incluirlas en sistemas de cultivo con fines de exportación, debido a la gran aceptación de frutas exóticas en los mercados internacionales. A pesar de esta riqueza y del gran potencial de estas especies, muchas de ellas todavía se encuentran en estado silvestre, semisilvestre, o en proceso de domesticación (CRFG, 2007). La mayoría de estas frutas aún no se han consolidado en los sistemas de producción del país; por esta razón, el crecimiento como cultivo es lento y a veces negativo, debido a muchas de índole genética y ambiental. Para el caso del tomate de árbol, durante los años 2000 y 2009, el desarrollo del cultivo en Colombia decreció en $0,5 \%$ anual en cuanto a producción y rendimiento (Agronet, 2010), sin un incremento significativo del área sembrada el mismo periodo.

Colombia cuenta con ofertas ambientales óptimas para el cultivo del tomate de árbol, pero aún no se ha hecho uso de su variabilidad genética. Por lo tanto, los agricultores se ven enfrentados a problemas de diferente índole, entre ellos los fitosanitarios, que no permiten explotar el potencial genético de la fruta. Además, este último está determinado por la variabilidad genética y su adaptación a condiciones tropicales (Tafur, 2006).

Jarvis et al. (2005) indican que la distribución de las especies está, en cierta medida, determinada por el ambiente. Así, según la distribución de estos factores ambientales (clima, suelo, relieve) en el espacio, se puede establecer si la especie está presente o no. Sin embargo, esta se ve afectada por su condición semisilvestre y por la falta de soporte tecnológico, dado que el desarrollo de estas especies como cultivo ha sido fundamentado sobre el conocimiento empírico, fruto del esfuerzo de los agricultores (Tafur, 2006).

Uno de los instrumentos que se usan actualmente para identificar áreas potenciales de colecta de germoplasma son los sistemas de información geográfica (SIG), los cuales permiten obtener una visión más clara sobre la distribución de una especie. Berger et al. (2003) mencionan que se pueden identificar áreas potenciales para colecta, predecir la adaptación climática en otras áreas y comparar los diferentes tipos de adaptación climática.

A través del uso de mapas ambientales construidos y producidos bajo SIG, es posible estimar la probabilidad de encontrar una especie bajo determinadas condiciones ambientales y definir los sitios donde se encuentra determinada especie, y así optimizar recursos en la fase de colecta. Según Steiner y Greene (1996), esta metodología puede ser utilizada como un nuevo tipo de caracterización, adicional a los métodos tradicionales para la evaluación de germoplasma.

Recientemente, se han desarrollado programas de computación con base en SIG para llevar a cabo estudios de recursos genéticos, tales como FloraMap (Jones y Gladkov, 1999). Este programa fue desarrollado por el laboratorio GIS del Centro Internacional de Agricultura Tropical (CIAT), principalmente para la predicción de áreas de distribución de organismos en los hábitats naturales, cuando se conoce poco o nada de la ecología de la especie. Este programa ha sido utilizado antes con éxito en la predicción geográfica de especies de Passiflora en cinco países andinos (Segura et al., 2003) así como para definir el lugar más apropiado para la colección núcleo de Phaseolus en América (Tohme et al., 1995).

FloraMap asume que el clima en los puntos de observación o de colección de una especie, es representativo de la gama ambiental del organismo y es usado como referencia para la calibración y generación de un modelo, el cual es utilizado para evaluar la probabilidad de otros sitios que son convenientes para la especie.

El programa provee diagramas climáticos de zonas ambientales similares donde se encuentra 
la especie, además de un mapa con una probabilidad superficial que proporciona el potencial climático donde el organismo podría existir; sin embargo no toma en cuenta factores como suelo, pendiente del terreno, brillo solar y vientos entre otros. Para estimar la posible distribución de C. betacea en el departamento de Nariño, se realizó un mapa de distribución general utilizando los datos de origen de todas las poblaciones de la colección (Jones y Gladkov, 1999).

La Universidad de Nariño cuenta con una colección de trabajo de 56 introducciones documentadas de C. betacea, provenientes de las zonas productoras del departamento de Nariño. A partir de la información de los datos de pasaporte de esta población, los objetivos de este trabajo fueron establecer las zonas eco-climáticas donde se ubica su variabilidad y definir otras zonas, donde potencialmente se puede encontrar la especie.

\section{MATERIALES Y MÉTODOS}

Con la información de los datos de pasaporte de la colección de trabajo de tomate de árbol del grupo de investigación en Producción de Frutales Andinos (GPFA) de la Universidad de Nariño, se construyó una matriz con la identidad de las 56 introducciones, su procedencia, altura sobre el nivel del mar y coordenadas geográficas de colecta o sitio de selección. Posteriormente, con esta matriz se elaboró una base de datos en una hoja de cálculo Excel, con las siguientes variables: latitud y longitud (en sistema decimal), y altitud, para un total de 56 puntos. Luego, los datos fueron exportados en formato Dbase IV (*.dbf) para ser analizados con el sistema de información geográfica FloraMap (Jones y Gladkov, 1999).

El programa FloraMap es un sistema para pronosticar la distribución o las posibles zonas de adaptación para organismos naturales cuando se conoce poco o nada sobre la fisiología detallada del organismo. Se asume que el clima en los puntos de colección de un conjunto de individuos es representativo del rango de ambientes en que habita el organismo. El clima en los puntos de colección se usa para orientar la calibración cuando se genera un modelo de probabilidad del clima, el cual es utilizado para evaluar la probabilidad de otros puntos geográficos que son convenientes para la especie (Jones y Gladkov, 1999).

Para el caso de América Latina y África, FloraMap usa una rejilla de 10 min del arco (cerca de $18 \mathrm{~km}$ en el ecuador). Las rejillas son estimadas a partir de la interpolación de 10.000 estaciones meteorológicas ubicadas en Latinoamérica. El programa utiliza un algoritmo basado en el cuadrado inverso de la distancia entre las cinco estaciones más cercanas y el punto interpolado. Se utilizan 36 variables climáticas, 12 promedios mensuales para temperatura, precipitación, e intervalo de temperatura diurna. La precipitación y el intervalo de temperatura diurna permanecen independientes de la altitud (Jones, 1991).

También se realizo un Análisis de Componentes Principales (ACP) para determinar las relaciones entre la variación climática y la variación en la distribución geográfica de C. betacea. Con base en el ACP, se agruparon las zonas climáticas, empleando como criterio de clasificación el método de Ward.

\section{RESULTADOS Y DISCUSIÓN}

En el análisis de componentes principales se encontró que los dos primeros componentes explican $96,82 \%$ de la variabilidad climática, en la que se encuentran creciendo los genotipos de la colección de trabajo de tomate de árbol de la Universidad de Nariño. En la figura 1 se observa en forma general la distribución posible de $C$. betacea en el departamento de Nariño, donde los sitios de colección, están representados por los puntos negros, que se distribuyen a lo largo de la zona alto andina.

Se determinaron cinco zonas climáticas con diferentes niveles de probabilidad de encontrar po- 


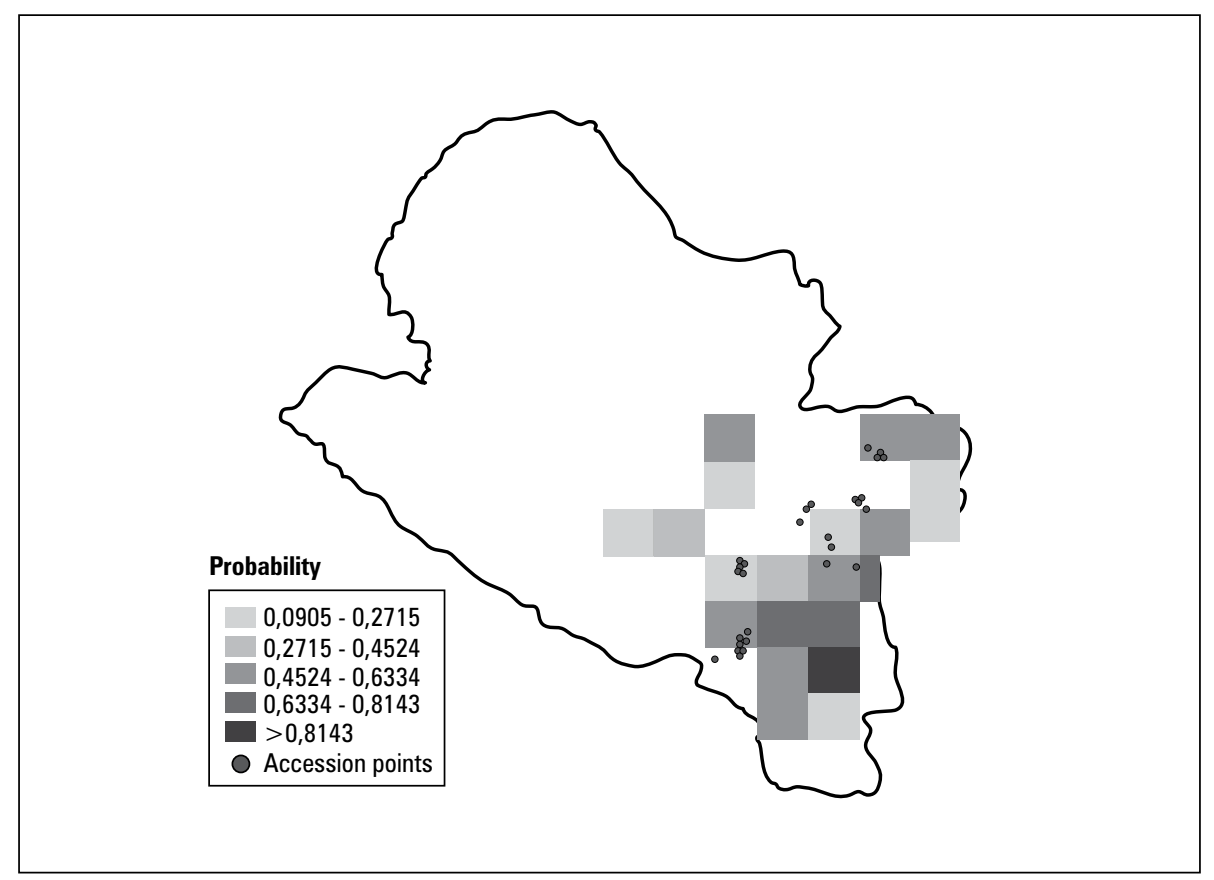

Figura 1. Puntos de origen de las 56 poblaciones de Cyphomandra betacea y su distribución general estimada en el departamento de Nariño.

blaciones de $C$. betacea, dadas por gradientes en la tonalidad del gris mostrado en la figura 1.

Los cuadrantes más oscuros indican mayor probabilidad, en este caso superior a $81,43 \%$, de tener ambientes adecuados para esta especie, localizados en regiones de los municipios de Funes, Puerres, Córdoba y Potosí, con una temperatura promedio de $13,2^{\circ} \mathrm{C}$ y una precipitación anual de $1.088 \mathrm{~mm}$.

Regiones de los municipios de Pasto, Contadero, Cumbitara, Los Andes, San Pablo, Cólon, Belén, La Unión, Albán y San Pedro de Cartago presentan una probabilidad entre 63,44 y $81,43 \%$ (figura 1). Esta zona exhibe una temperatura promedio anual de $17,6^{\circ} \mathrm{C}$ y una precipitación de 1.575 $\mathrm{mm}$. Otras regiones de los municipios de Buesaco, Iles, Gualmatan, Pupiales e Ipiales, con una temperatura promedio anual de $13^{\circ} \mathrm{C}$ y precipitación de $998 \mathrm{~mm}$ muestran probabilidades que van desde $45,24 \%$ hasta $63,64 \%$ de encontrar la especie objeto de estudio. La probabilidad más baja de encontrar $C$. betacea está entre 27,15 y 45,24\% y corresponde a los municipios de Santacruz, Túquerres, Tangua y Yacuanquer en zonas cuya temperatura y precipitación promedio anual son de $12,8^{\circ} \mathrm{C}$ y $1.188 \mathrm{~mm}$, respectivamente.

\section{Análisis de clasificación (AC)}

El criterio de clasificación de Ward permitió agrupar introducciones que se desarrollan en zonas climáticas similares pero sin ser excluidas por un dato no común en el grupo. A partir del análisis de componentes (AC) del ACP de las 56 introducciones de $C$. betacea, se identificaron dos grupos climáticos. En el primero se incluyeron 35 puntos, los cuales están a una altura comprendida entre 2.280 y 2.938 msnm, el segundo incluyó 21 puntos con una elevación entre 2.028 y 2.760 msnm (figura 2).

Los dos grupos climáticos presentan perfiles similares (figura 3), comportamiento que, según Núñez et al. (2008), hace sospechar que no existe un posible centro de distribución de C. betacea. para el departamento de Nariño. Estos grupos no son contrastantes en cuanto a la temperatura media y la precipitación pluvial. Esto se corrobora con la similitud que tienen, en cuanto al 


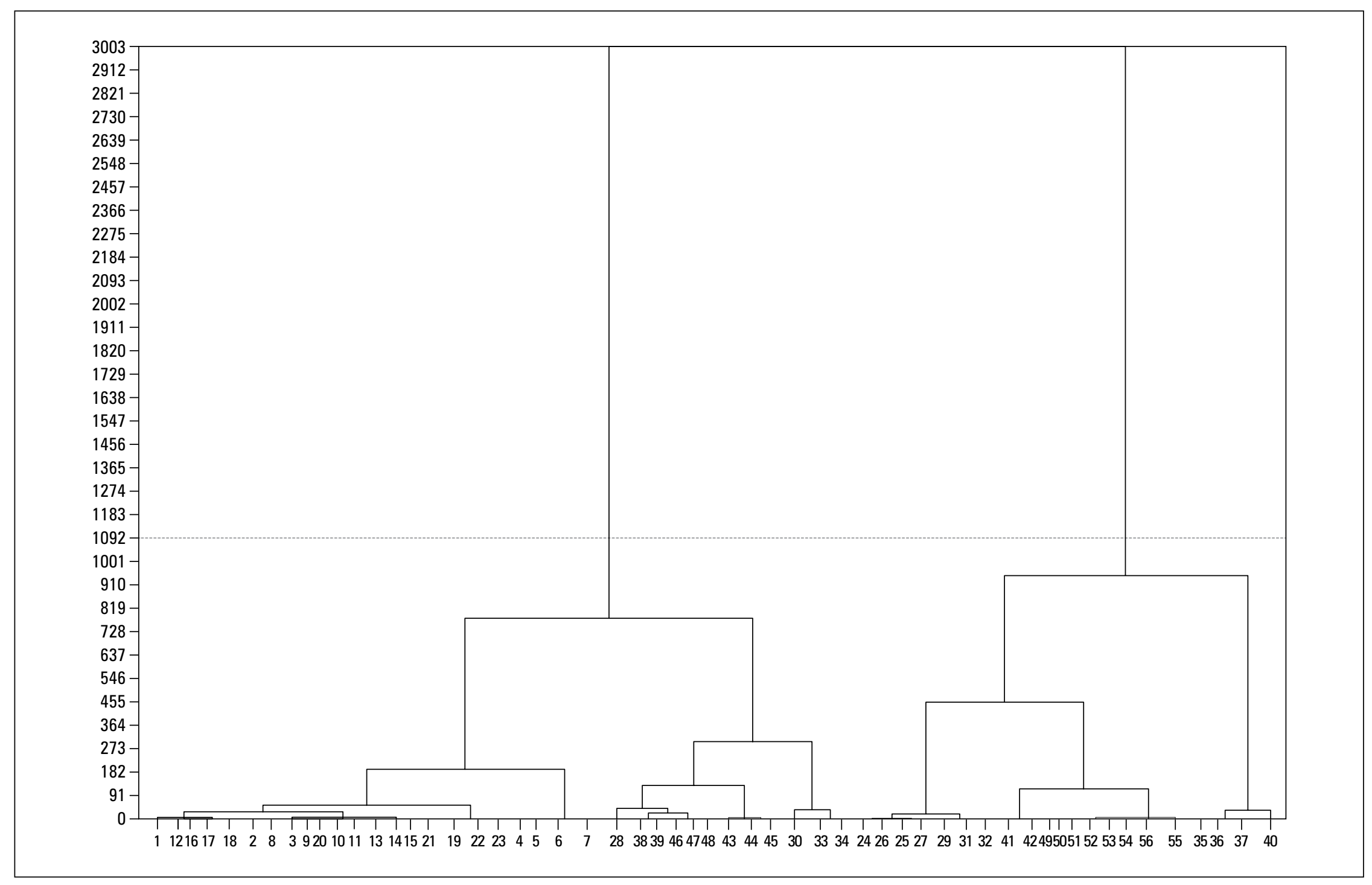

Figura 2. Dendograma de 56 genotipos de Cyphomandra betacea formado a apartir del ACP de las variables climáticas estimadas. 
Encontrados 35 puntos; elevación 2.500,1 msnm

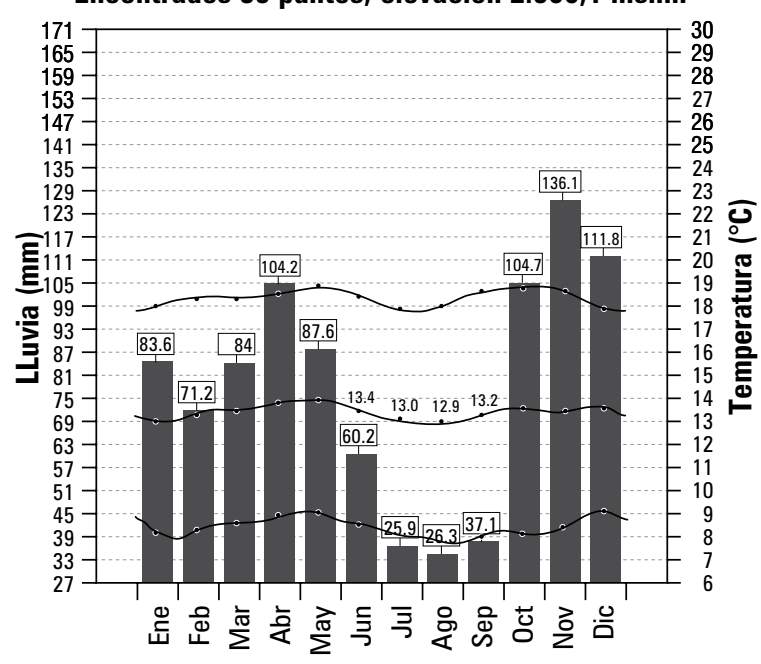

Encontrados 21 puntos; elevación 2.318,6 msnm

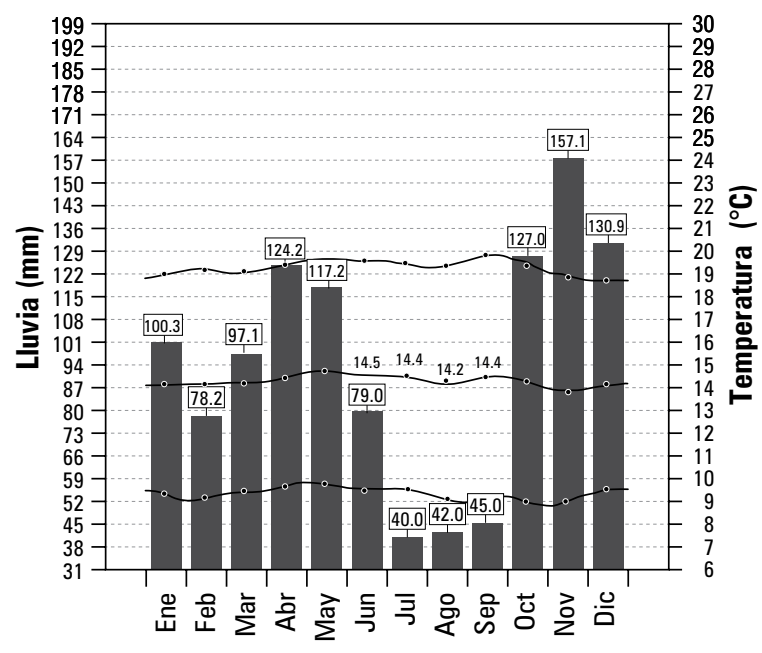

Figura 3. Diagramas climatológicos de los dos grupos climáticos obtenidos mediante el AC del ACP de las 56 introducciones de Cyphomandra betacea del departamento de Nariño.

comportamiento climático anual. Para el caso de la precipitación, este es de tipo bimodal, con dos picos altos, uno a finales del primer trimestre y otro en el transcurso del último trimestre de cada año y con los puntos más bajos en los meses de julio, agosto y septiembre, con valores que oscilan entre 924 y $1.124 \mathrm{~mm}$. De la misma manera, la temperatura promedio durante todo el año en los grupos encontrados fluctúa entre los 13,27 y $14,43^{\circ} \mathrm{C}$ para los grupos uno y dos, en su orden (figura 4). De acuerdo con la similitud climática expuesta por los dos grupos y según la escala de clasificación de Köppen, estos corresponden a un clima tipo $\mathrm{Cb}$, denominado clima templado húmedo, mesotérmico (García, 1988).

En la figura $5 \mathrm{~A}$ se muestran las probabilidades de encontrar introducciones de C. betacea en condiciones ecoclimáticas similares a las del grupo 1 del AC. Los mayores valores de probabilidad (mayores a 0,81 ) se presentaron en algunas zonas de Buesaco y el sur de Pasto. Regiones de Pupiales, Gualmatan, Iles, Córdoba, Contadero y Funes muestran probabilidades que van desde 0,63 hasta 0,80 . Valores entre 0,45 y 0,62 se en- cuentran en el norte de Pasto y sectores ubicados en Puerres y Potosí. Algunas zonas de Ipiales, Cuaspud, Guachucal, Sapuyes y Túquerres mostraron las menores probabilidades $(0,09-0,44)$.

La probabilidad de encontrar accesiones de C. betacea en condiciones eco-climáticas similares a las del grupo dos del AC es superior a 0,81 en zonas de Cumbitara, Los Andes y Santacruz, desde 0,63 hasta 0,80 en regiones de La Unión, Colón, San Pablo, San Bernardo, Albán, San Pedro de Cartago, Arboleda, Tangua y Funes, y entre 0,45 y 0,62 en algunas localidades de La Cruz, Linares, El Tambo, Samaniego y La Llanada. La probabilidad comprendida entre 0,27 y 0,44 se encuentra en algunas partes de Mallama y algunos sectores del norte de Cumbal, por último y en menor probabilidad de 0,09 a 0,27 en sitios localizados en Ricaurte, Sandona, La Florida, Yacuanquer y algunas localidades situadas al sur del municipio de Cumbal (figura 5B).

Lo anterior concuerda con lo consignado por el Ministerio de Agricultura y Desarrollo Rural, la Corporación Colombia Internacional y la Secre- 


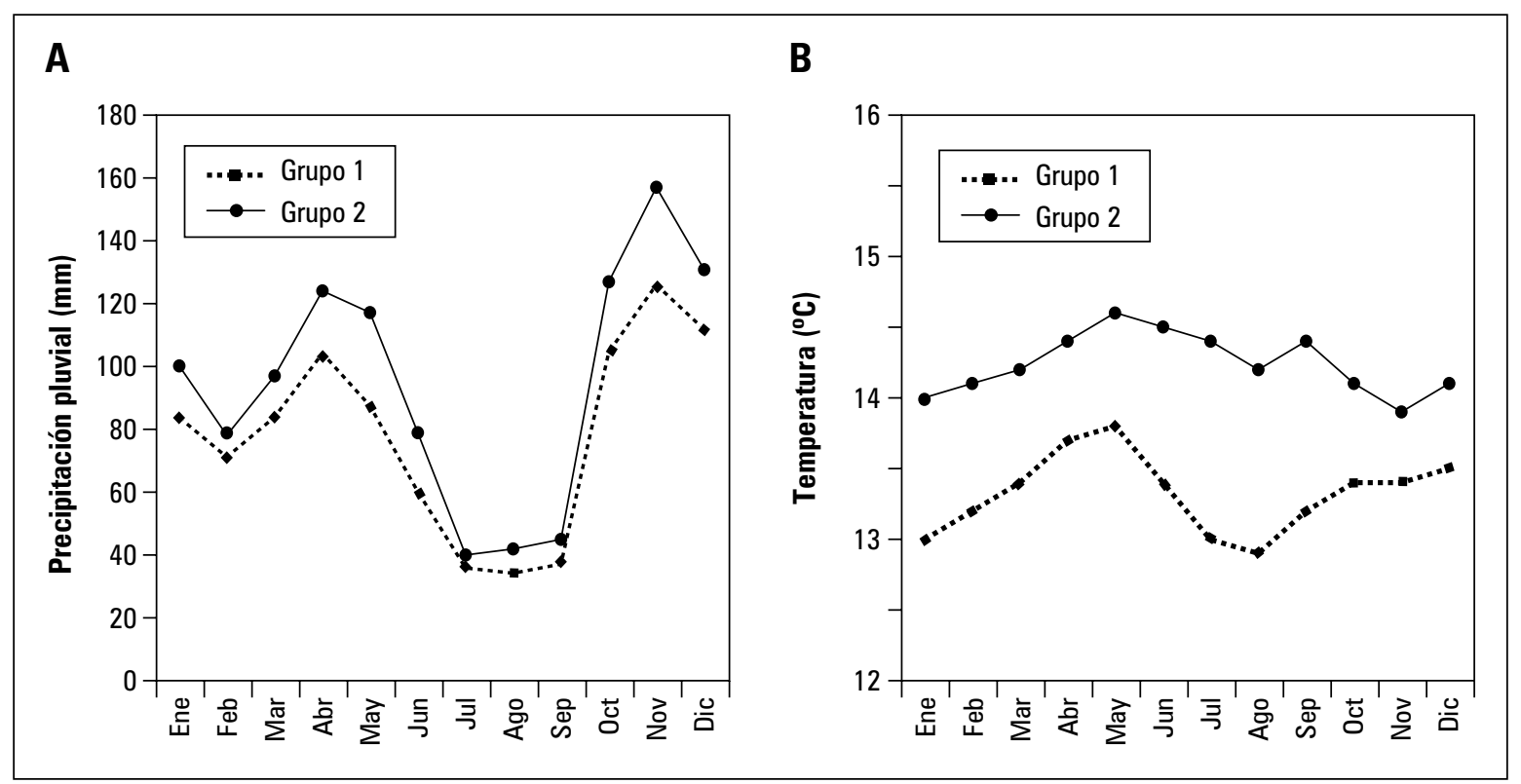

Figura 4. Comparación de la precipitación pluvial mensual (A) y la temperatura media mensual (B) en las dos posibles zonas de distribución de Cyphomandra betacea en Nariño.

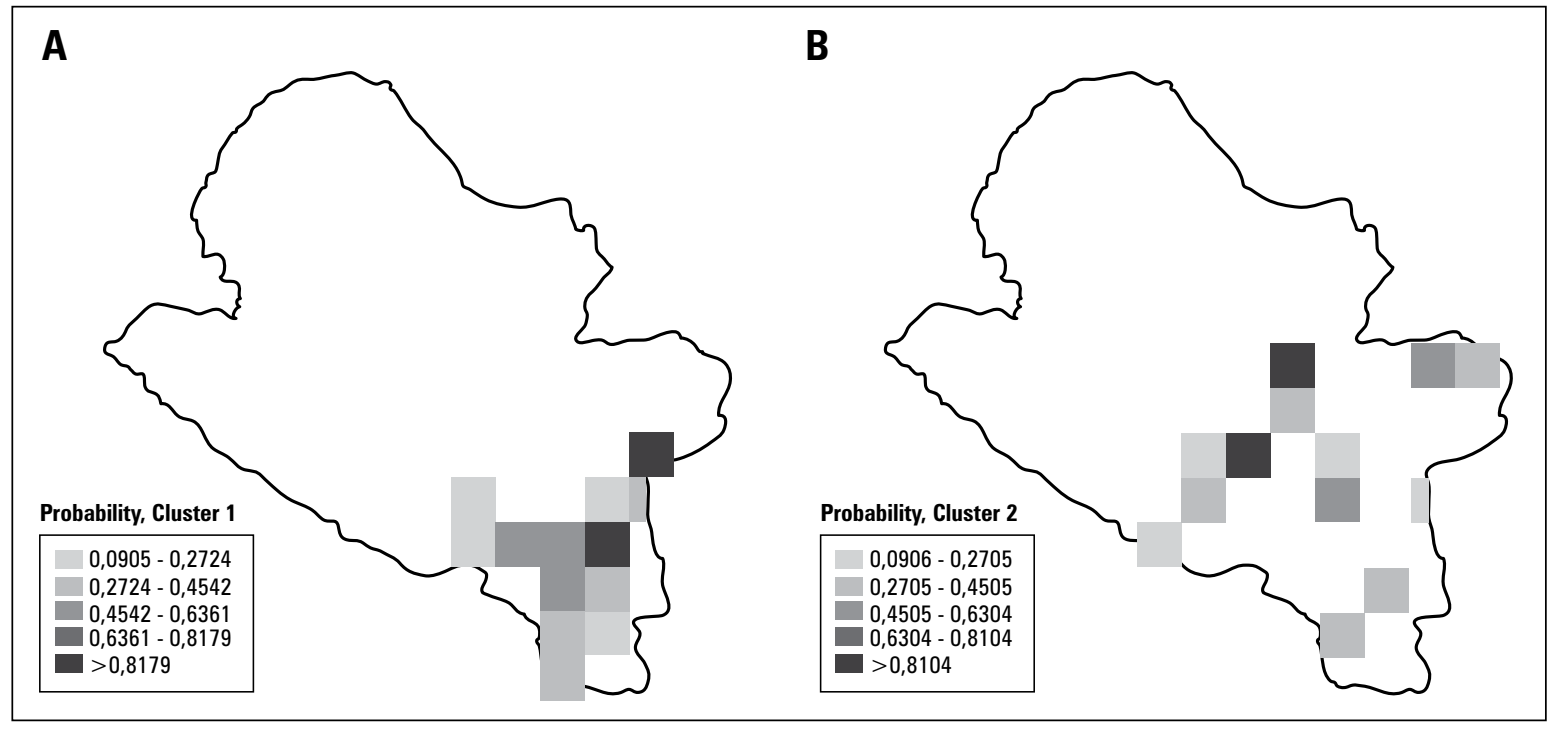

Figura 5. Distribución estimada de grupos climáticos 1 (A) y 2 (B) donde se distribuyen las introducciones de Cyphomandra betacea en Nariño.

taría de Agricultura y Medio Ambiente de Nariño (2010), que establecen que para 2008 en el departamento se encontraban establecidas alrededor de 580 ha cultivadas con C. betacea, localizadas en su mayoría en los municipios representativos de los dos grupos climáticos del AC, indicando que en estos sitios y algunos, donde la especie no se encuentra en estado cultivado, es- taría distribuida la variabilidad de Nariño, siendo sitios potenciales de colecta de germoplasma.

\section{CONCLUSIONES}

La distribución de $C$. betacea en el departamento de Nariño se encuentra en la región alto andina, 
en zonas de relieve montañoso en alturas que oscilan entre 1.500 y 3.000 msnm y pluviosidad entre 400 y $2.500 \mathrm{~mm}$ promedio anual. Se encontraron dos grupos climáticos donde se distribuye tomate de árbol, los cuales no permiten establecer un centro de distribución. Dentro de los dos grupos climáticos, el grupo uno estuvo conformado por introducciones provenientes del sur y las del grupo dos por aquellas provenientes del centro y norte del departamento de Nariño.

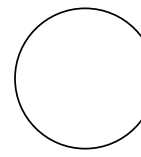

Agronet. Estadísticas de Productos Agropecuarios. Ministerio de Agricultura y Desarrollo Rural. Republica de Colombia. En: http://www.agronet. gov.co/agronetweb, consulta: febrero de 2010.

Berger, J., J. Abbo y N.C. Turner. 2003. Ecogeography of annual wild cicer species. The poor state of the world collection. Crop Sci. 43(2), 1076-1090.

Bohs, L. 1988. Ethnobotany of the genus Cyphomandrea (Solanaceae). Economic Bot. 43 (2), 143-163.

California Rare Rruit Growers (CRFG) 2007. Tamarillo (Cyphomandra betacea (Cav.) Sendt.) Solanaceae. En: http://www.crfg.org/pubs/ff/tamarillo.html; consulta: marzo de 2007.

García, E. 1988. Modificaciones al sistema de clasificación climática de Köppen, para adaptarlo a las condiciones de la República Mexicana. Instituto de Geografía, Universidad Nacional Autónoma de México, México.

Jarvis, A., M.E. Ferguson, D.E. Williams, L. Guarino, P.G. Jones, H.T. Stalker, J.F. Valls, R.N. Pittman, C.E. Simpson y P. Bramel. 2003. Biogeography of wild Arachis: assessing conservation status and setting future priorities. Crop Sci. 43(3), 1100-1108.

Jarvis, A., S. Yeamam, L. Guarino y J. Tohme. 2005. The role of geographic analysis in locating, understanding and using plant genetic diversity. Methods Enzymol. 395, 279-298.

Jones, P.G. 1991. The CIAT climate database Version 3.41. Machine readable dataset. Centro Internacional de Agricultura Tropical (CIAT), Cali, Colombia.

Jones, P.G. y A. Gladkov. 1999. FloraMap: a computer tool for predicting the distribution of plants and the other organisms in the wild, version 1.0. En: Jones, A.L. (ed.). Centro Internacional de Agricultura Tropical (CIAT), Cali, Colombia.

Lobo, M. 2006. Recursos geneticos y mejoramiento de frutales andinos: una visión conceptual. Ciencia Tecnol. Agropec. 7 (2), 40-54.

\section{REFERENCIAS BIBLIOGRÁFICAS}

Ministerio de Agricultura y Desarrollo Rural (MADR), Corporación Colombia Internacional (CCI) y Secretaria de Agricultura y Medio Ambiente de Nariño. 2010. Consolidado Agropecuario 2009. Secretaría de Agricultura y Medio Ambiente de Nariño, Pasto, Colombia.

Morineau, A. 1998. SPAD, Version 3. Manuel de Prise en main. Cisia-Ceresta, Saint Mande, Francia.

Núñez, C.A., R. Nieto, A.F. Barrientos, S. Segura, J. Sahagún y F. Gonzales. 2008. Distribucion y caracterizacion eco-climatica del genero Crategus L. (Rosaceae, Subfam. Maloideae) en México. Revista Chapingo Serie Horticultura 14(2), 177-184.

Pringle, G. y B. Murray. 1991. Interspecific hybridization involving the tamarillo, Cyphomandra betacea (Cav.) Sendt. (Solanaceae). J. Crop Hort. Sci. 19,103-111.

Segura, S., G. Cooppens, L. Lopez, M. Grum y L. Guarino. 2003. Mapping the distribution of five species of Passiflora in Andean countries. Genetics Resources and Crop Evolution 50(6), 555-566.

Steiner, J.J. y S.L. Greene. 1996. Proposed ecological descriptors and their utility for plant germplasm collections. Crop Sci. 36, 439-451.

Tafur, R. 2006. Propuesta frutícola para Colombia y su impacto en la actividad económica, nacional, regional y departamental. pp. 47-68. En: Memorias Primer Congreso Colombiano de Horticultura. Sociedad Colombiana de Ciencias Hortícolas, Bogotá.

Tohme, J., P. Jones, S. Beebe y M. Iwanaga, 1995. The combined use of agroecological and characterization data to establish the CIAT Phaseolus vulgaris core collection. pp. 95-115. En: Odgkin, T., A.H.D. Brown, T.J.L. Van Hintum y E.A.V. Morales (eds.). Core collection of plant genetic resources. International Plant Genetic Resources Institute (IPGRI), Rome.

Valencia, R. y O. Torres. 2011. Distribución del tomate de árbol Cyphomandra betacea (Cav.) Sendt. y caracterización eco-climática en las zonas del cultivo de Nariño. Trabajo de grado. Facultad de Ciencias Agrícolas, Universidad de Nariño, Pasto, Colombia. 